\title{
APRENDIZAGEM ATIVA: DESENVOLVIMENTO DE APLICATIVO PARA CÁLCULO DA FATURA MENSAL DE ENERGIA ELÉTRICA
}

DOI: 10.37702/2175-957X.COBENGE.2021.3504

Laura Lisiane Callai dos Santos - laura.callai.santos@gmail.com

Universidade Federal de Santa Maria

Rua Ernesto Barros 1345

96506-322 - Cachoeira do Sul - RS

Thales Loebens - thalesloebenst@gmail.com

Universidade Federal de Santa Maria

RUA JULIO DE CASTILHOS

95800-000 - Venâncio Aires - RS

Celso Becker Tischer - cbtischer@gmail.com

Universidade Federal de Santa Maria

Rua Ernesto Barros 1345

96506-322 - Cachoeira do Sul - RS

Matheus Dottes Binttencourt - matheusdottes@gmail.com

Universidade Federal de Santa Maria

Rua Ângelo Torineli 78

89259-200 - Jaraguá do Sul - SC

Resumo: A aprendizagem ativa permite que o estudante tenha uma intervenção ativa no seu próprio processo de aprendizagem. Este artigo apresenta o desenvolvimento de um aplicativo que define, em termos econômicos, qual a melhor modalidade tarifária para o consumidor de Baixa Tensão aderir utilizando a Aprendizagem Baseada em Problema (PBL). A tarifa convencional possui o mesmo preço de tarifa ao longo do dia, já a Tarifa Branca possui valores diferenciados de tarifa ao longo dia, buscando diminuir o carregamento de energia no horário de ponta, onde o preço da tarifa é mais caro. Durante o dia, a tarifa branca possui três valores distintos: ponta, intermediário e fora de ponta. Os finais de semana e feriados contam com apenas um valor fixo durante todo o dia. $O$ aplicativo foi desenvolvido no MIT App Inventor, desenvolvido para a plataforma Android se mostrando como uma ferramenta informativa e educacional que vai viabilizar a escolha do consumidor em optar pela tarifa convencional ou branca. Se observou 


\section{COBENGE

uma aprendizagem dos conteúdos utilizados, bem como da linguagem de programação, contribuindo na formação profissional e autônoma dos alunos.

Palavras-chave: Aprendizagem Ativa. Aplicativo. Tarifa de Energia Elétrica. Aprendizagem Baseada em Problemas 


\section{APRENDIZAGEM ATIVA: DESENVOLVIMENTO DE APLICATIVO PARA CÁLCULO DA FATURA MENSAL DE ENERGIA ELÉTRICA}

\section{INTRODUÇÃO}

De acordo com as novas Diretrizes Curriculares Nacionais do Curso de Graduação em Engenharia, a utilização de metodologias para aprendizagem ativa dos estudantes deve ser estimulada (BRASIL, 2019). De acordo com Elmôr Filho (2019) é necessário valorizar atividades de aprendizagem em que os conceitos possam ser abordados e desenvolvidos a partir de investigações baseadas na intuição e bom senso, em vez de definições abstratas.

Existem diferentes estratégias e métodos de aprendizagem ativa, sendo que a Aprendizagem Baseada em Problema (Problem-based learning - PBL) vem sendo vastamente estudada e empregada.

Esse método consiste no aprendizado no contexto de problemas reais, onde os estudantes constroem seus conhecimentos buscando respostas.

A PBL tem como objetivo estimular os estudantes a desenvolver a iniciativa e criatividade, desenvolver o pensamento crítico, promover o trabalho em equipe, desenvolver a capacidade de comunicação e relacionar conteúdos interdisciplinares de forma integrada (ALVES ET AL., 2007).

Considerando as vantagens da PBL, foi proposto a alunos do Curso de Engenharia Elétrica do Campus da Universidade Federal de Santa Maria em Cachoeira do Sul (UFSMCS) o desenvolvimento de um aplicativo para smartphone que realiza o cálculo da fatura mensal de energia elétrica para consumidores do Grupo B. A proposta vem pela verificação da demanda por estudos sobre a tarifa branca, principalmente quanto a viabilidade econômica da mudança tarifária desses consumidores.

Diante do exposto, este trabalho apresenta o estudo e execução da proposta, originados pela aplicação da PBL, que resultou no desenvolvimento de uma ferramenta intuitiva que auxilia consumidores de baixa tensão na escolha da melhor tarifa, bem como informa aos consumidores a diferença financeira entre as tarifas vigentes. A ferramenta foi desenvolvida através do MIT App Inventor sendo possível analisar diferentes perfis de consumo para consumidores do Grupo B (residencial, comercial e rural).

Para a consolidação da proposta, os conceitos relacionados com estrutura tarifária brasileira, cálculo do consumo de energia elétrica e criação de aplicativos, foram buscados pelos acadêmicos envolvidos.

\section{ESTRUTURA TARIFÁRIA BRASILEIRA}

Segundo a Agência Nacional de Energia Elétrica (ANEEL), órgão responsável por fiscalizar e regulamentar a energia elétrica no Brasil, as unidades consumidoras de energia elétrica são classificadas em dois grupos: A e B. De acordo com a Resolução Normativa (RN) n‥ 414 da ANEEL (2010a), o Grupo A é composto por unidades consumidoras com fornecimento em tensão igual ou superior a 2,3 kV, ou atendidas a partir de sistema subterrâneo de distribuição em tensão secundária, caracterizado pela tarifação binômia. Já o Grupo B é composto por unidades consumidoras com fornecimento em tensão inferior a 2,3 kV, caracterizado pela estruturação tarifária monômia.

Para os consumidores do Grupo B, é aplicada a tarifa monômia de fornecimento que, segundo a RN №. 479 da ANEEL (2012), é constituída por valor monetário aplicável 
unicamente ao consumo de energia elétrica ativa, obtida pela conjunção da componente de demanda de potência e de consumo de energia elétrica que compõem a tarifa binômia. $A$ tarifa monômia é composta pela soma de dois componentes: Tarifa de Uso do Sistema de Distribuição (TUSD), a qual se refere ao valor monetário unitário determinado pela ANEEL, utilizado para efetuar o faturamento mensal de usuários do sistema de distribuição de energia elétrica pelo uso do sistema. A outra componente refere-se a Tarifa de Energia (TE), utilizado para efetuar o faturamento mensal referente ao consumo de energia.

A tarifa convencional monômia é a estrutura tarifaria mais utilizada entre os consumidores do grupo $\mathrm{B}$, devido ao seu longo tempo de implantação. Essa tarifa é caracterizada por consumo com valor único, independentemente do horário do dia. Devido as características dessa tarifa, às cargas elétricas residenciais aumentam de forma significativa em determinado horário do dia, chamado de período de ponta. Como a rede elétrica trabalha sobrecarregada no período de ponta, encontrou-se na tarifa branca, uma forma de diminuir as cargas nesse período, buscando o deslocamento das cargas para períodos de fora ponta.

\section{$2.1 \quad$ Tarifa Branca}

Até o ano de 2018, os consumidores de baixa tensão não obtinham alternativas de tarifação, ou seja, eram faturados de uma única forma, na qual a tarifa era única independentemente do horário do dia. Em 2018, a ANEEL criou a tarifa branca, que reflete o uso de energia elétrica de acordo com o horário consumido. Dessa forma, o consumidor pode alterar o seu horário de consumo para o período de fora ponta e reduzir seus gastos com energia elétrica, além de melhorar o fator de utilização das redes elétricas.

A tarifa branca é dividida em três períodos: ponta, intermediário e fora de ponta. Feriados e finais de semana são considerados períodos de fora ponta durante todo o dia.

- Horário de ponta: período diário de 3h consecutivas, com exceção feita aos sábados, domingos e feriados nacionais;

- Horário intermediário: período de horas conjugadas ao horário de ponta, aplicados exclusivamente às unidades consumidoras que optem pela Tarifa Branca. Pode variar de $1 \mathrm{~h}$ à 1 h30 antes e depois do horário de ponta; e

- Horário fora de ponta: período diário composto pelas horas consecutivas e complementares ao horário de ponta e intermediário.

O horário de ponta é normalmente definido pelas concessionárias entre $18 \mathrm{~h}$ até às 21h. O período intermediário é a hora imediatamente anterior e pela hora posterior ao período de ponta. Já o horário fora ponta é todos os horários que não seja o período de ponta e intermediário.

A Figura 1 compara, em porcentagem de $\mathrm{R} \$ / \mathrm{MWh}$, as Tarifas Convencional e Branca. Nota-se que no período de fora ponta a tarifa branca é vantajosa para o consumidor, enquanto no período de ponta a tarifa possui um valor muito elevado. A Tarifa Convencional se mantém constante ao longo de todos os horários do dia. 
Figura 1 - Comparativo entre a Tarifa Branca e Tarifa Convencional

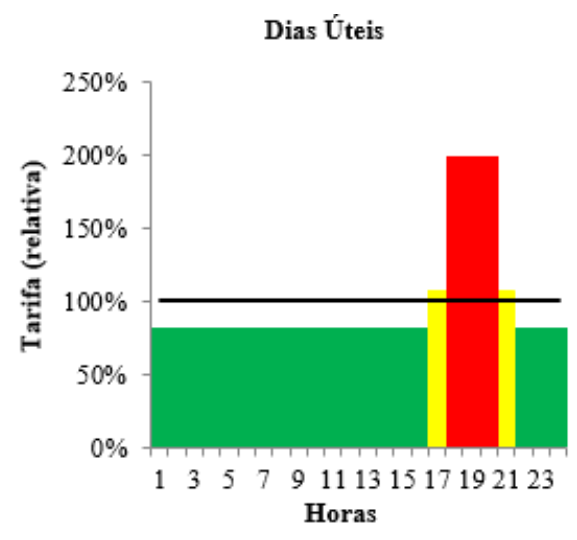

(a)

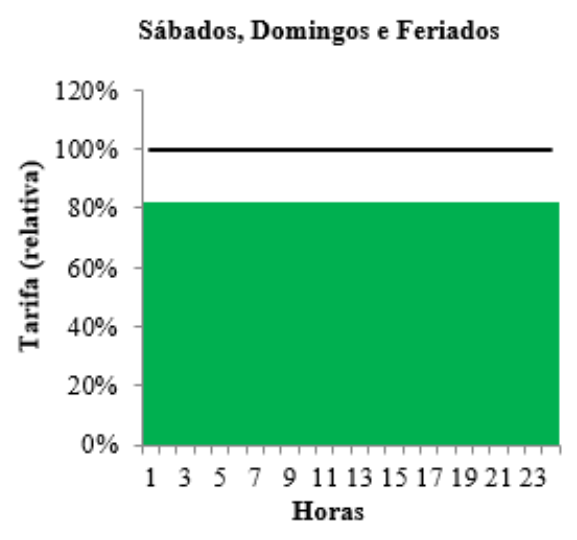

(b)

Conforme Resolução Normativa ํ․ .733/2016 da ANEEL, a tarifa branca começou a ser aplicada a partir de 1ㅇ de janeiro de 2018 para consumidores com média anual de consumo superior a $500 \mathrm{kWh} / \mathrm{mês.} \mathrm{Em} 1^{\circ}$ de janeiro de 2019, foi a vez dos consumidores com média anual de consumo superior a $250 \mathrm{kWh} /$ mês optarem por adquirir a nova modalidade tarifária. Desde $1^{\circ}$ de janeiro de 2020 , todas as unidades consumidoras podem migrar para a tarifa branca.

Caso o consumidor verifique benefícios na adesão à tarifa branca, a solicitação deverá ser feita à concessionária de energia. Após a análise do pedido, a concessionária tem até 30 dias para realizar a troca do medidor de energia, sendo o custo de aquisição e instalação do novo medidor responsabilidade da concessionária. Se o consumidor não perceber a vantagem da tarifa branca ele pode solicitar junto a concessionária a volta da tarifa convencional. Para que o consumidor não tenha que tomar a decisão de aderir a tarifa branca sem saber se terá benefícios econômicos, a utilização de ferramentas digitais tornase vantajosa. Dessa forma, foi desenvolvido um aplicativo que define qual a melhor modalidade tarifária para o consumidor de baixa tensão, de acordo com os seus hábitos de consumo utilizando o MIT APP INVENTOR.

\section{MIT APP INVENTOR}

O App inventor é um software de código aberto criada pela Google, e mantida pelo Massachusetts Institute of Technology (MIT), que permite desenvolver aplicativos Android usando um navegador da Web. Através do MIT, é possível criar aplicativos selecionando componentes e montando blocos, especificando como o aplicativo deve se comportar. A criação do aplicativo é feita de forma visual, juntando peças como se fosse um quebracabeça.

As aplicações do App inventor podem ser instaladas em qualquer telefone Android ou tablet. Caso o usuário não possuir um telefone Android, poderá simular o aplicativo desenvolvido com um emulador Android diretamente do seu computador. A Figura 2 demonstra o funcionamento do App inventor. 
Figura 2 - Programação MIT App Inventor

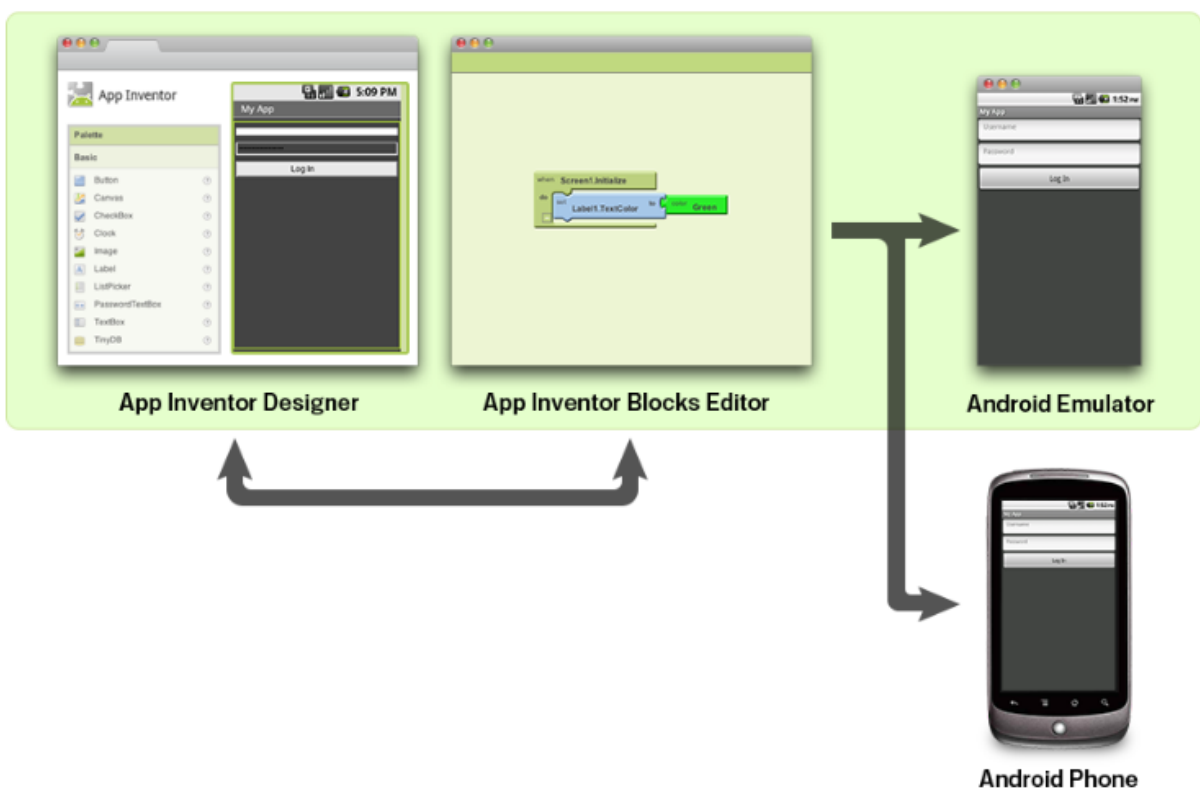

O App inventor possui uma linguagem de programação relativamente fácil, tornando acessível a entrada de alunos no mundo da programação Android. Com a popularidade da ferramenta, muitas pessoas estavam usando o App Inventor para criar aplicativos mais complexos, com recursos limitados. Desta forma, o App Inventor vem sendo remodelado, transformando-se em uma ferramenta mais profissional.

\section{ESTRUTURA DO APLICATIVO}

A tarifa branca é uma nova opção que o consumidor de baixa tensão tem de reduzir sua fatura mensal de energia elétrica. Antes de optar pela nova tarifa é importante o solicitante conhecer o seu perfil de consumo. Se o consumidor costuma utilizar a energia elétrica nos períodos fora de ponta, a adesão a tarifa branca poderá ser conveniente. No entanto, se o consumo for maior nos períodos de ponta e intermediário é recomendado ao consumidor continuar com a tarifa convencional. A mudança para a nova tarifa exige cuidados, visto que a escolha precipitada pode trazer aumento na conta de energia elétrica. Desse modo, é importante que o consumidor realize uma simulação do seu consumo de energia no aplicativo antes de optar pela tarifa branca. A Figura 3 apresenta a estrutura do aplicativo. 
Figura 3 - Estrutura do aplicativo

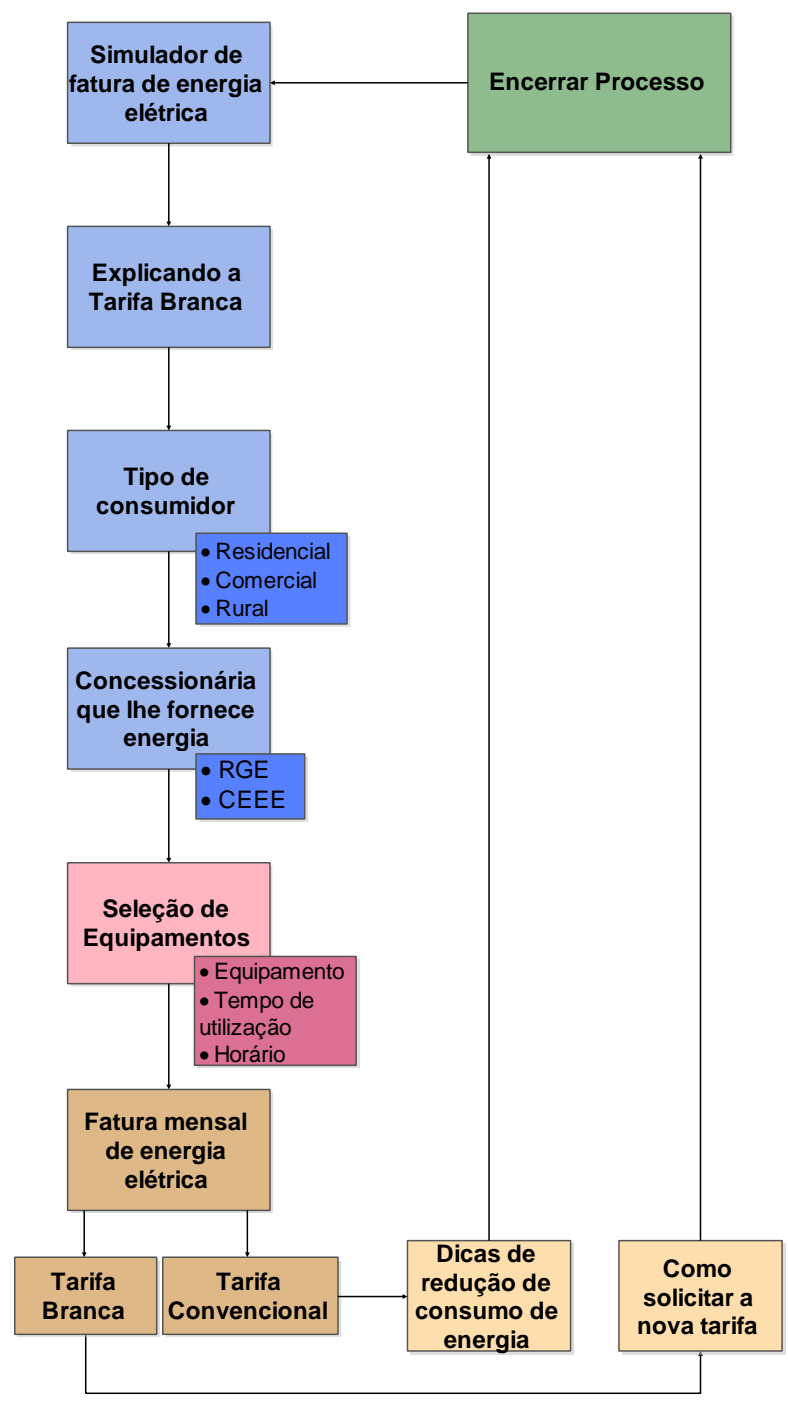

Conforme apresentado na Figura 3 o consumidor poderá simular a sua conta de energia elétrica e verificar qual tarifa é mais vantajosa.

O aplicativo está dividido em três etapas, sendo elas:

- Etapa 1: Inicialização;

- Etapa 2: Seleção de equipamentos;

- Etapa 3: Resultados.

\subsection{Etapa 1: Inicialização}

$\mathrm{Na}$ etapa de inicialização é mostrado a tela inicial do aplicativo, com o título: "Simulador de Fatura de Energia Elétrica". Na Figura 4 essa tela é demonstrada. 
Figura 4 - Tela de início do Aplicativo

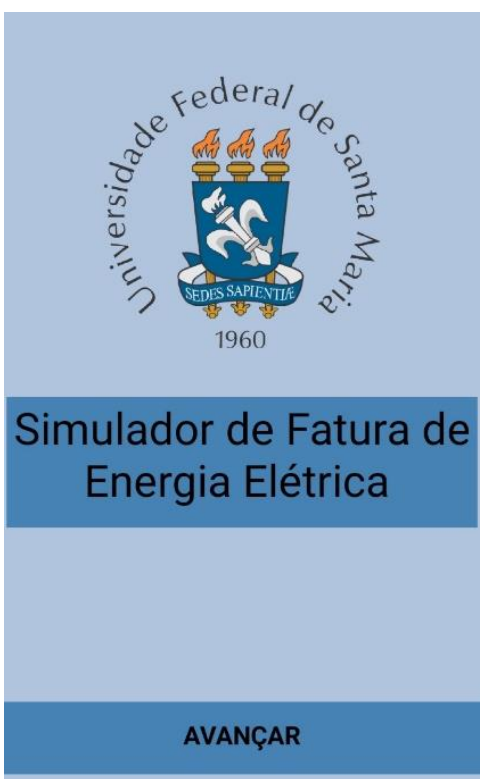

Fonte: Elaborado pelos Autores (2019)

Ao clicar no botão "Avançar" uma nova aba é aberta, Figura 5. Essa tela explica ao consumidor o que é a tarifa branca, quais suas vantagens e horários dos períodos de ponta, intermediário e fora ponta.

Figura 5 - Tela explicativa sobre a nova tarifa

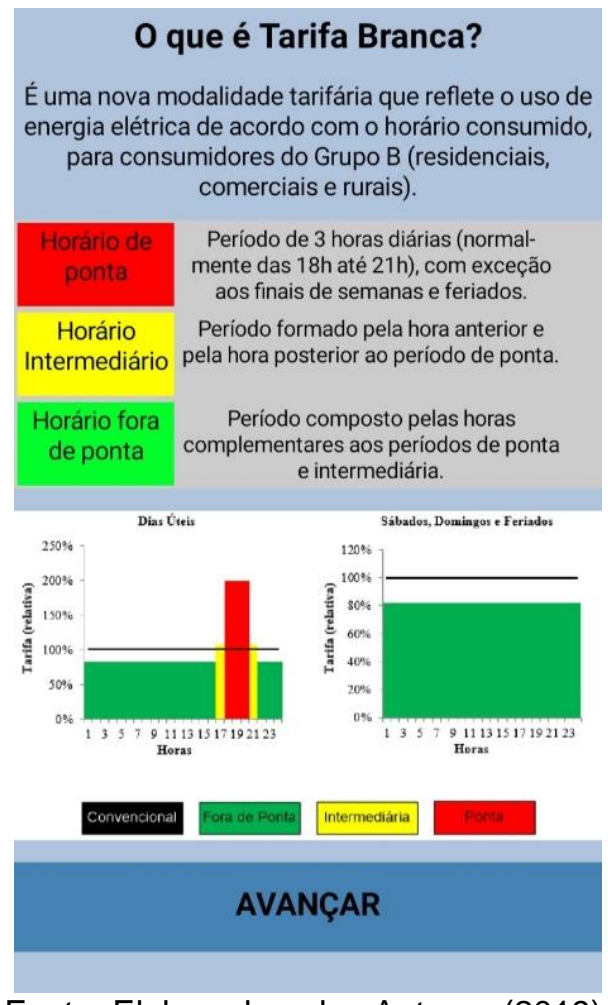


Em seguida, o consumidor deve selecionar qual o subgrupo de consumo. Dentre as opções estão: Residencial, comercial ou rural. A Figura 6 mostra a tela de seleção.

Figura 6 - Tela de seleção do subgrupo de consumo

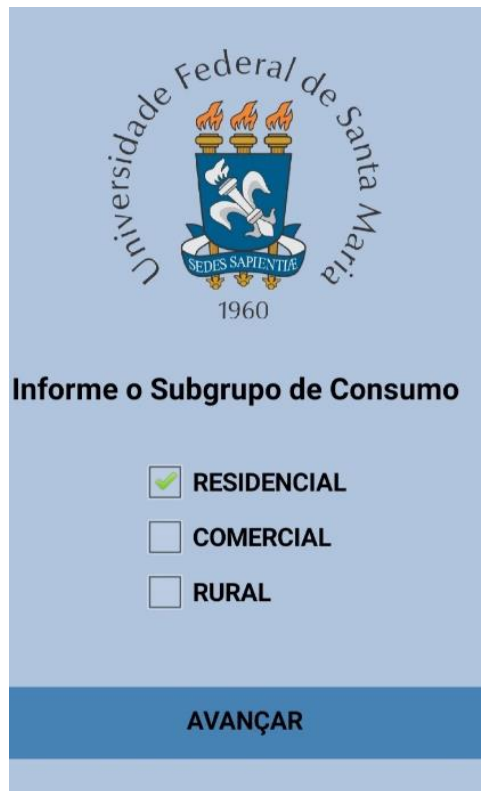

Fonte: Elaborado pelos Autores (2019)

Realizadas tais operações, o consumidor deverá informar qual concessionária the fornece energia, Figura 7. Dentre as opções estão: Companhia Estadual de Energia Elétrica (CEEE) e Rio Grande Energia (RGE). Ambas concessionárias estão localizadas no estado do Rio Grande do Sul, realizando a distribuição de energia ou atuando como supridoras para concessionárias menores.

Figura 7 - Tela de seleção da concessionária de energia elétrica

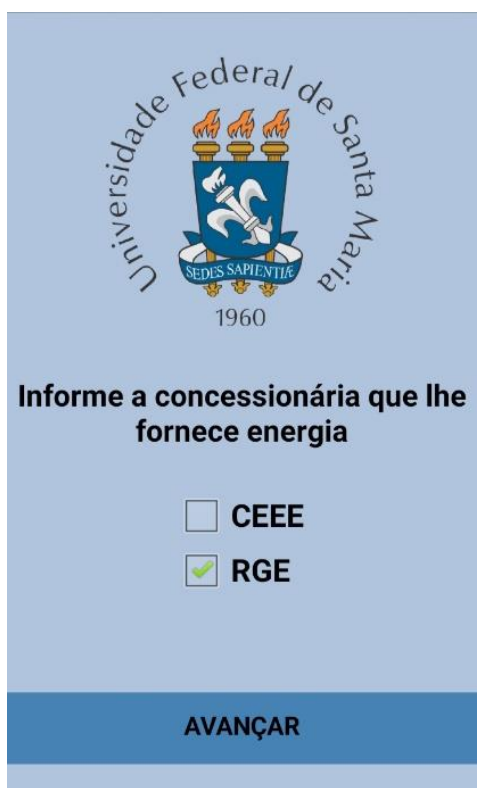

Fonte: Elaborado pelos Autores (2019). 
Após o consumidor informar o subgrupo e a concessionária, a ferramenta definirá os valores da Tarifa pelo Uso do Sistema de Distribuição (TUSD) e Tarifa de Energia (TE), em $\mathrm{R} \$ / \mathrm{kWh}$, nas tarifas convencional e branca. Para obter os valores das tarifas, considerouse que a bandeira tarifária de energia é verde, ou seja, condições favoráveis de geração de energia, sem nenhum acréscimo na tarifa. Os impostos da fatura de energia elétrica não foram considerados nos cálculos.

$\mathrm{Na}$ próxima etapa da estrutura do aplicativo o usuário seleciona os aparelhos elétricos e eletrônicos que possui em sua residência. Através dos aparelhos selecionados é possível obter o custo mensal com energia elétrica.

\subsection{Etapa 2: Seleção de equipamento}

Nesta etapa será fornecida uma lista de equipamentos para que o usuário selecione os eletroeletrônicos e eletrodomésticos que possui em sua residência ou comércio. Caso o usuário possuir algum equipamento que não contenha na lista, o mesmo poderá ser incluído ao catálogo.

Deverá ser informado junto ao aparelho que o consumidor possuir: a quantidade do mesmo, a potência em Watts e o tempo de uso do aparelho nos períodos de fora ponta, intermediário e ponta. $\mathrm{Na}$ ferramenta desenvolvida, a lista de aparelhos elétricos e eletrodomésticos está separada em 5 telas diferentes. A Figura 8 demonstra a primeira tela da lista de aparelhos.

Figura 8 - Tela Lista de Aparelhos Elétricos

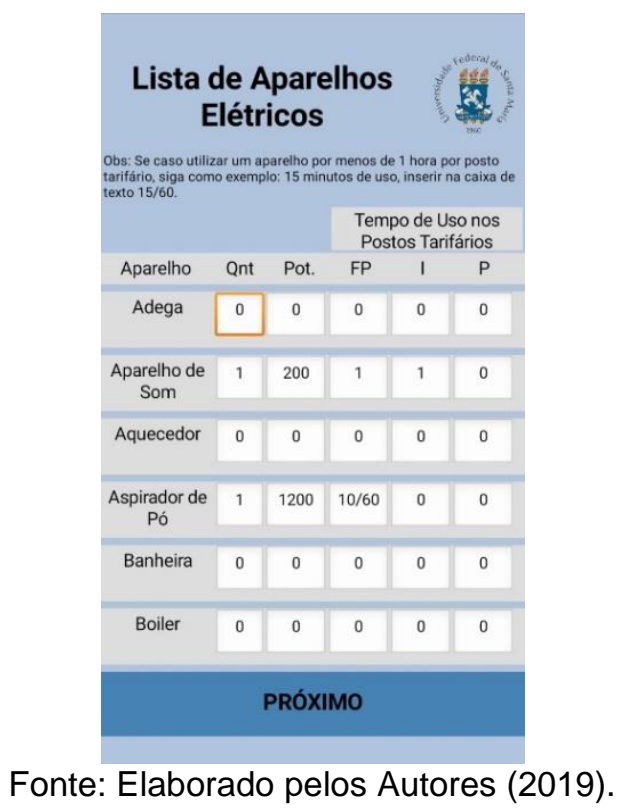

Inseridos os aparelhos e o seu tempo de uso nos postos tarifários, a próxima etapa da estrutura do aplicativo é realizar o cálculo do consumo mensal de energia, juntamente com as faturas de energia considerando as tarifas convencional e branca.

\subsection{Etapa 3: Resultados}

Através do cálculo do consumo total mensal, pode-se calcular os valores da fatura considerando a tarifa branca e a tarifa convencional. O aplicativo informará também, em termos financeiros, qual a modalidade mais adequada para o consumidor aderir. Os resultados das tarifas de energia podem ser observados na "Tela Resultados" como mostra a Figura 9. 
Figura 9 - Tela resultados

\begin{tabular}{|c|c|}
\hline \multicolumn{2}{|c|}{$\begin{array}{c}\text { Fatura Mensal de Energia } \\
\text { Elétrica }\end{array}$} \\
\hline \multicolumn{2}{|c|}{ Consumo Total (Kwh/mês): 1025.70} \\
\hline Tarifa Convencional: & $\mathrm{R} \$ 574.67$ \\
\hline Tarifa Branca: & $\mathrm{R} \$ 568.48$ \\
\hline \multicolumn{2}{|c|}{$\begin{array}{l}\text { MODALIDADE TARIFÁRIA MAIS } \\
\text { ADEQUADA: }\end{array}$} \\
\hline \multicolumn{2}{|c|}{ Tarifa Branca } \\
\hline
\end{tabular}

Fonte: Elaborado pelos Autores (2019).

A ferramenta desenvolvida também traz sugestões ao consumidor em mudar o seu hábito de consumo com o deslocamento de cargas para períodos em que a energia é mais barata, bem como recomendações de troca de aparelhos que consomem uma elevada potência, buscando uma maior eficientização energética, como exemplo a troca de lâmpadas incandescente e fluorescentes por LED.

\section{CONSIDERAÇÕES FINAIS}

Este trabalho apresentou a utilização da metodologia PBL para o desenvolvimento de um aplicativo que determina a viabilidade econômica da utilização da tarifa branca para consumidores residenciais do grupo B. A utilização da PBL como metodologia de aprendizagem ativa apresentou um retorno positivo, pois foi necessário através do estudo, desenvolver um projeto em equipe que contribui para o estímulo de competências técnicas, sociais, empreendedoras e pessoais dos acadêmicos envolvidos.

Além disso, resultou na aprendizagem de criação do aplicativo, associada ao estudo da metodologia de cálculo de consumo de energia elétrica para diferentes modalidades tarifárias. Com isso, se trabalhou atributos que são essenciais para o futuro engenheiro, como a resolução de problemas, apresentação de resultados, planejamento, organização e criatividade.

\section{REFERÊNCIAS}

Nota Técnica no 311/2011. SRE-SRD/ANEEL - Estrutura tarifária para o serviço de distribuição de energia elétrica. ANEEL, 2011.

Resolução Normativa no 414/2010, 2010a.

Resolução Normativa no 733/2016, $2016 a$.

Resolução Normativa no 479/2012, 2012.

Tarifa Branca, 2019a. Disponível em: <http://www.aneel.gov.br/tarifa-branca>.

ALVES, A.; MOREIRA, F.; SOUZA, R. O papel dos tutores na aprendizagem baseada em projectos: três anos de experiência na Escola de Engenharia da Universidade do Minho. 
In: BARCA, A. et al. 2007.

BRASIL. Resolução nำ 2, de 24 de abril de 2019. Institui as Diretrizes Curriculares Nacionais do Curso de Graduação em Engenharia. Diário Oficial da União, Ministério da Educação/Conselho de Educação/Câmara de Educação Superior. Brasília, DF, 26 abr. de 2019. Ed. 80. Seção 1, p. 43.

ELMÔR FILHO, Gabriel et al. Uma Nova Sala de Aula é Possível. 1ª ed. Rio de Janeiro: LTC, 2019.

FIGUEIRÓ, I. C. A tarifa horária para os consumidores residenciais sob o foco das redes elétricas inteligentes - REI. 2013, 127 f. Dissertação (Mestrado em Engenharia Elétrica) - Santa Maria: Universidade Federal de Santa Maria, 2013.

KNAK NETO, N. Metodologias para modelagem de cargas de baixa tensão considerando a integração de resposta à demanda, Geração Distribuída e Veículos Elétricos. 2017, 237 f. Tese (Doutorado em Engenharia Elétrica) - Universidade Federal de Santa Maria.

\section{LAMIN, H. Medição Eletrônica em Baixa Tensão: Aspectos Regulatórios e}

Recomendações para implantação. 2009. 184 f. Dissertação (Mestrado em Engenharia Elétrica) - Universidade de Brasília, Brasília, 2009.

OLIVEIRA, C. T. A. DE. Desenvolvimento de ferramenta para análise de migração de consumidores de baixa tensão à tarifa branca. 2017. Dissertação (Bacharel em Engenharia Elétrica) - Santo Ângelo: Universidade Regional Integrada do Alto Uruguai e das Missões.

SANTOS, L. L. C. DOS. Metologia para análise da tarifa branca e da geração distribuída de pequeno porte nos consumidores residenciais de baixa tensão. 2014 , 91 f. Dissertação (Mestrado em Engenharia Elétrica) - Santa Maria: Universidade Federal de Santa Maria.

\section{ACTIVE LEARNING: DEVELOPMENT OF APPLICATION TO CALCULATE MONTHLY ELECTRICITY}

Abstract: Active learning allows the student to have an active intervention in his own learning process. This article presents the development of an application that defines, in economic terms, which is the best tariff modality for Low Voltage consumers to join using Problem Based Learning (PBL). The conventional tariff has the same tariff price throughout the day, whereas the White Tariff has different tariff values throughout the day, seeking to reduce the energy loading during peak hours, where the tariff price is more expensive. During the day, the white tariff has three distinct values: peak, intermediate and off-peak. Weekends and holidays have a fixed amount throughout the day. The application was developed on the MIT App Inventor, developed for the Android platform, showing itself as an informative and educational tool that will enable the consumer to choose the conventional or white tariff. Learning of the contents used, as well as of the programming language, was observed, contributing to the students' professional and autonomous training.

Keywords: Active learning. Application. Electricity Tariff. Problem-based learning. 